\title{
Wildnisbildung als Möglichkeit zur Professionalisierung zu- künftiger Geographielehrer/innen - ein neuer Ansatz zur Bildung für nachhaltige Entwicklung?
}

\author{
*anne.lindau@geo.uni-halle.de, Martin-Luther-Universität Halle-Wittenberg \\ ** daniela.hottenroth@gmail.com, Staatliche Regelschule Gotthold-Ephraim-Lessing Nordhausen \\ ${ }^{* * *}$ martin.lindner@biodidaktik.uni-halle.de, Martin-Luther-Universität Halle-Wittenberg
}

eingereicht am: 01.02.2016, akzeptiert am: 27.04.2016

\begin{abstract}
Der in den deutschen Nationalparken seit mehreren Jahren thematisierte und praktizierte Ansatz der Wildnisbildung stellt möglicherweise einen neuen Zugang zur Bildung für nachhaltige Entwicklung (BNE) in der universitären Lehrer/innenbildung im Fach Geographie dar. Am Beispiel des DBU-geförderten Projektes „Wildnis macht stark“ wird eine Konzeption für die Umsetzung von Wildnisbildung im Rahmen des Geographie-Lehramtsstudiums vorgestellt und mit ersten Forschungsergebnissen zur Wirksamkeit in der Praxis gestützt, die auf die Potenziale des Ansatzes hinweisen.
\end{abstract}

Keywords: Wildnisbildung, Bildung für nachhaltige Entwicklung, Lehrer/innenbildung, Geographie, Lehrer/innenprofessionalisierung

\section{Wilderness education as an option for pre-service teacher training in geography - a new concept in education for sustainable development}

In recent years wilderness education has developed as a new educational concept in national parks. Potentially wilderness education is seen as a cutting-edge approach for education in sustainable development (ESD) in pre-service teacher training at universities, esp. for geography. In cooperation with Harz National Park, the project Wilderness makes you strong was developed, inter alia for teacher training. This article demonstrates the implementation of wilderness education within teacher training for geography and presents initial research results for its effectiveness in practice.

Keywords: Wilderness education, education for sustainable development, teacher training, geography, professionalization of teachers

\section{Einleitung}

Das Gesicht Mitteleuropas ist geprägt durch Zivilisation. Seit der Jungsteinzeit wurde die Landschaft kontinuierlich in eine Kulturlandschaft umgewandelt, in der von Menschen unbeeinflusste Gebiete wenig Raum haben (Zucchi \& Stegmann 2006). Dennoch steigt die Zahl der in Europa lebenden Wildtiere stetig, was unter anderem auf ein Umdenken hinsichtlich der Landnutzung sowie der Unterschutzstellung von Gebieten zurückzuführen ist. So sind Wolf und Luchs zwei Arten, die auch in Deutschland als Wildtiere wieder größere Gebiete erobern - zwei Erfolgsgeschichten der aktuellen Wildnisdebatte.

Allmählich wächst jedoch das Bewusstsein dafür, sich selbst überlassenen Flächen durch das Konzept des Prozessschutzes wieder mehr Beachtung zu schenken. Prozessschutz bedeutet, dass alle natürlichen Prozesse vom Menschen ungestört zugelassen werden, auch auf bisher anthropogen geprägten Standorten. Diese natürlichen Prozesse führen schließlich zur Entwicklung von Wildnis, wenn auch zu einer Sekundärwildnis. Wildnisgebiete bieten Rückzugsorte für regionalspezifische Flora und Fauna und dienen somit der Bewahrung von Biodiversität (Bittner 2014).

In den letzten Jahren entstanden zahlreiche Bildungsinitiativen, um u. a. Schülerinnen und Schülern Zugang zu verwildernden Räumen zu ermöglichen. Ziel dieser Bildungsmaßnahmen ist es, die Akzeptanz für die Genese dieser Räume zu erhöhen. Daneben bietet Wildnisbildung auch zahlreiche Anknüpfungspunkte, nachhaltiges Denken und Handeln zu entwi- 
ckeln. Die Ziele solcher Bildungsprogramme können z. B. in Wildniscamps, wie u. a. im Nationalpark Harz durchgeführt, umgesetzt werden. Wildnisbildung wird hier für unterschiedliche Zielgruppen angeboten, um die Idee dieses Konzepts zu verbreiten (Langenhorst \& Lude 2014).

Der Artikel hat zum Ziel, Wildnisbildung als einen möglichen Ansatz für die universitäre Lehrer/innenbildung für das Fach Geographie vorzustellen und zu diskutieren. Dazu werden am Beginn des Artikels die Begriffe der Wildnis und Wildnisbildung sowie die Ziele und bisherigen Konzeptentwicklungen aus Sicht der Nationalparke und Bundesregierung präsentiert. Im weiteren Verlauf werden Ansatzpunkte der Wildnisbildung für die universitäre Geographie-Lehrer/innenbildung kritisch diskutiert. Mithilfe von zwei Interventionsstudien werden Ergebnisse zu Potenzialen und Grenzen von Wildnisbildung aufgezeigt. Innerhalb der vorzustellenden Forschungsansätze wird folgender Fragestellung nachgegangen: Inwieweit bietet Wildnisbildung als Teil von Bildung für nachhaltige Entwicklung einen Ansatz für die universitäre Lehrer/innenbildung?

\section{Potenziale der Wildnisbildung für die universitäre Lehrer/innenbildung}

\subsection{Zum Wildnisbegriff}

Während der Wildnisbegriff für Nordamerika durch spezifische Kriterien klar definiert wird, erweist sich eine Begriffsdefinition in Europa gegenwärtig noch als schwierig. Die Weltnaturschutzunion „International Union for Conservation of Nature" (IUCN) definiert Wildnis bezogen auf seine Fläche (in einer deutschen Übersetzung durch EUROPARC Deutschland) wie folgt:

\section{Wildnisdefinition auf internationaler Ebene (IUCN)}

„Schutzgebiete der Kategorie Ib [Wildnisgebiete i. e. S.] sind i. d. R. ausgedehnte ursprüngliche oder (nur) leicht veränderte Gebiete, die ibren natürlichen Charakter bewahrt haben, in denen keine ständigen oder bedeutenden Siedlungen existieren; Schutz und Management dienen dazu, den natürlichen Zustand zu erhalten" (EUROPARC Deutschland 2010, 19).

Auf europäischer Ebene wurde 2012 von der „European Wilderness Working Group" eine Arbeitsdefinition in Zusammenhang mit einer "Wilderness Strategy" der Europäischen Union formuliert, die ebenfalls den Fokus auf ursprüngliche Wildnis durch die Erhaltung von Wildnisgebieten in Europa legt.
Wildnisdefinition auf europäischer Ebene (European Wilderness Working Group)

„Wildnisgebiete sind große, unveränderte oder nur leicht veränderte Naturgebiete, die von natürlichen Prozessen beherrscht werden und in denen es keine menschlichen Eingriffe, keine Infrastruktur und keine Dauersiedlungen gibt. Sie werden dergestalt geschützt und betreut, dass ihr natürlicher Zustand erhalten bleibt und sie Menschen die Möglichkeit $z u$ besonderen geistig-seelischen Naturerfahrungen bieten " [deutsche Übersetzung] (European Union 2012,10$)$.

Diese Ansätze gehen von der Vorstellung aus, dass es sich bei Wildnis um frei entwickelnde Landschaften handelt, die vom Menschen unberührt sind, wie sie teilweise noch heute in Teilen Kanadas, Sibiriens oder der Antarktis zu finden sind (Mittermeier et al. 2002).

Unvereinbar erscheinen jedoch diese Definitionen von Wildnis mit der Flächennutzung in Deutschland. So kann der Umfang der Kernwildnis in Europa mit zwei Prozent nur als rudimentär bezeichnet werden. Deshalb wurde zum Schutz dieser Gebiete für Deutschland eine vom Bundesamt für Naturschutz operable Begriffsdefinition für Wildnis entwickelt, welche die sekundäre Wildnis (vom Menschen ungenutzte Flächen, die jedoch durch menschliche Nutzung in der Vergangenheit geprägt sind) mit einschließt.

\section{Wildnisdefinition auf nationaler Ebene (Nationale Biodiversitätsstrategie) \\ „Wildnisgebiete i. S. der NBS [Nationale Biodi- versitätsstrategie] sind ausreichend große, (weitge- hend) unzerschnittene, nutzungsfreie Gebiete, die dazu dienen, einen vom Menschen unbeeinflussten Ablauf natürlicher Prozesse dauerhaft zu gewähr- leisten" (Finck et al. 2013, 343).}

Die Idee der Entwicklung sekundärer Wildnisgebiete in Deutschland hat ihren Ursprung in den Großschutzgebieten der Nationalparke der USA. Das nationale Strategiepapier zur biologischen Vielfalt des Bundesamtes für Umwelt, Naturschutz, Bau und Reaktorsicherheit (BMUB) formulierte erstmals 2007 das hohe und kontrovers diskutierte Ziel, bis 2020 zwei Prozent der Fläche Deutschlands in Wildnisgebiete zu überführen. Dies bedeutet, dass sich jene Landesflächen in Deutschland nach eigenen Gesetzmäßigkeiten entwickeln können und kein Eingriff in die natürliche Dynamik erfolgt. Als Gründe für diese Vision wird u. a. die Förderung der Biodiversität, die Zulassung von natürlichen Dynamiken und die Schaffung von Lebensräumen (z. B. Pioniervegetation, intakte Auenwälder) genannt (BMUB 2015). Aus diesen 
Gründen erweitert das BMUB die Entwicklung von zukünftigen nationalen Wildnisgebieten wie folgt:

„Um die natürlichen Prozesse der Lebensraumdynamik wieder zu aktivieren, muss ein bestimmter Flächenanteil Deutschlands von menschlicher Einflussnabme freigestellt werden. Dies betrifft vor allem die verbleibenden Reste der natürlichen Ökosysteme, kann jedoch auch Bereiche umfassen, die aus der menschlichen Nutzung fallen und sich künftig in Richtung einer "neuen Wildnis "[Sekundärwildnis, Anmerkung der Verfasser] hin entwickeln können"(Ebenda, 41).

In diesem Sinne existieren neben der Entwicklung von Wildnisflächen in Nationalparken auch Initiativen vom Bund für Umwelt und Naturschutz Deutschland (BUND) sowie von Stiftungen und Naturschutzverbänden außerhalb der Nationalparke, Wildnisgebiete einzurichten. So wurden 2013 zum Beispiel 16000 Hektar Naturerbeflächen vom BUND an die Deutsche Bundesstiftung Umwelt (DBU) übertragen, mit dem Ziel, in diesen Wildnisgebieten eine natürliche und sich selbst regulierende Entwicklung zu ermöglichen (DBU 2013).

Mit der Entstehung der vorwiegend durch Wald bedeckten sekundären Wildnisflächen gehen Bestrebungen einher, Bildungsmaßnahmen in diesen Schutzgebieten zu initiieren (BMUB 2015). Ziele dieser Bildungsmaßnahmen sind, die Bevölkerung für die Entstehung von Wildnisflächen und für Diskussionen zum Thema Wildnis zu sensibilisieren. Beispielhaft werden an dieser Stelle Initiativen der Nationalparke Bayerischer Wald (Projekt „Wildniscamp am Falkenstein“), Kellerwald-Edersee (Projekt „Waldscout - Wildnisexpedition“) und Harz (Projekt „Wildnis macht stark") genannt (Langenhorst et al. 2014). Ziel dieser Projekte ist es, Angebote zur Wildnisbildung zu schaffen, die Wildnis im Sinne von „Natur Natur sein lassen“ (Bibelriether 1998) und den Schutz dynamischer Prozesse in Nationalparken zum Inhalt haben. Ein zentrales Element der Wildnisbildung ist der zeitlich begrenzte Aufenthalt von Personen in Wildniscamps. Dabei handelt es sich um eine von Nationalparken für Bildungszwecke freigegebene Fläche, die mit unterschiedlichen Graden der infrastrukturellen Ausstattung aufwartet (BUND 2002).

Die Relevanz der Bestrebungen zum Thema Wildnis Bildungsangebote zu schaffen, zeigt auch das ausgeprägte Interesse an den Themen Natur und Wildnis in der Bevölkerung, wie die Naturbewusstseinsstudie 2013 des BMUB zeigt. Andererseits wird Wildnis als zivilisationsferner Ort und Erfahrungsraum von den Befragten wahrgenommen, der teilweise als bedrohlich oder gefährlich charakterisiert wird (BMUB \& BfN 2013; Langenhorst \& Lude 2014). Aufgrund der im Moment von der Bundesregierung angestrengten Bestrebungen, Wildnisentwicklung innerhalb und außerhalb von Nationalparken zu fördern sowie das Interesse der Bevölkerung an diesen Themen legen die Option nahe, Bildungsangebote für Schüler/innen zu schaffen, da gerade für diese Zielgruppe die Umsetzung der Wildnisstrategien eine Lebensbedeutsamkeit für die Zukunft aufweist. Gleichzeitig muss das Ziel von Lernangeboten zum Thema Wildnis sein, die teilweise kontroverse Debatte um die Vision der Bundesregierung, zwei Prozent der Fläche Deutschlands in Wildnisgebiete zu überführen, aufzunehmen. In diesem Rahmen stellt die universitäre Lehrer/innenbildung ein wichtiges Feld für die Anbahnung von Neuerungen im schulischen Umfeld dar, da zukünftigen Lehrkräften eine Funktion als Multiplikatorinnen und Mulitplikatoren hinsichtlich der Verbreitung der Thematik Wildnis zukommen kann.

\subsection{Wildnisbildung als Ansatz einer Bildung für nachhaltige Entwicklung}

Bildung für nachhaltige Entwicklung ist ein von den Vereinten Nationen unterstütztes, weltweites Bildungskonzept, das in der Schule und in der Lehrer/innenbildung einen wichtigen Baustein darstellt.

„BNE befähigt Lernende, informierte Entscheidungen zu treffen und verantwortungsbewusst zum Schutz der Umwelt, für eine bestandsfähige Wirtschaft und einer gerechten Gesellschaft für aktuelle und zukünftige Generationen zu handeln und dabei die kulturelle Vielfalt zu respektieren. Es geht um einen lebenslangen Lernprozess, der wesentlicher Bestandteil einer hochwertigen Bildung ist. BNE ist eine ganzheitliche und transformative Bildung, die die Lerninhalte und-ergebnisse, Pädagogik und die Lernumgebung berücksichtigt. Ihr Ziel/Zweck ist eine Transformation der Gesellschaft" (DUK 2014, 12).

Das Ziel einer Bildung für nachhaltige Entwicklung ist der Austausch und das Reflektieren über einen ökologischen und ökonomisch verantwortbaren Umgang mit Ressourcen der Erde sowie Rohstoffen und Energie. Aus sozialer Sicht geht es um einen gerechten Umgang der Menschen miteinander. Die Idee des Bildungskonzeptes einer Bildung für nachhaltige Entwicklung ist es, dass Menschen befähigt werden, das gemeinsame Leben auf dieser Erde so zu gestalten, dass eine lebenswerte Gegenwart und Zukunft für alle möglich wird (De Haan 1999; Stoltenberg 2013).

Innerhalb dieser Anforderungen kommt insbesondere der Lehrer/innenbildung aufgrund der zukünf- 
tigen Funktion als Multiplikatorinnen und Multiplikatoren eine bedeutende Rolle zu. Um der Forderung einer Bildung für nachhaltige Entwicklung gerecht zu werden, muss sich die Lehrer/innenbildung in allen Bereichen an deren Leitbild orientieren (LeNa 2014). Die Standards für die Lehrer/innenbildung im Bereich Bildungswissenschaften formulieren die Anforderungen innerhalb der Lehrer/innenprofessionalisierung, die sowohl die fachlichen, fachdidaktischen und pädagogischen Anforderungen an die zukünftigen Lehrkräfte umfassen (KMK 2004) und das Leitbild einer Bildung für nachhaltige Entwicklung in sich integriert (LeNa 2014).

Der Ansatz der Wildnisbildung (im Sinne der Schaffung von Bildungsangeboten zum Thema Wildnis) wird aus der Perspektive der Nationalparke aufgrund seiner ganzheitlichen und systemischen Charakteristik als Möglichkeit der Integration in das Konzept einer Bildung für nachhaltige Entwicklung gesehen.

„Wildnisbildung ist durch die handlungsorientierte Verknüpfung von Naturerleben und ökologischer Bildung anhand konkreter Wildnis-Phänomene mit dem Fokus der Reflexion des Verbältnisses von Mensch und Natur charakterisiert. Hierbei spielen die Nachhaltigkeits-Perspektiven Partizipation, Vernetzung, Mehrdimensionalität (Nachhaltigkeits-Dreieck Ökonomie - Ökologie - Soziales), globaler Blick und Generationenfolge eine zentrale Rolle. Die Wildnisbildung versteht sich als Teil der Bildung für nachhaltige Entwicklung (BNE)“ (Langenhorst 2012, o.S.).

Wildnisbildung in den Nationalparken verfolgt in erster Linie das Ziel der Akzeptanzbildung in der Bevölkerung gegenüber der Existenz und Entstehung von Wildnisgebieten. „Neben dem Zulassen der Eigendynamik von Natur ist es daher die größte Herausforderung, den Menschen den Zugang zur unmanipulierten Natur zu ermöglichen und Respekt und Verständnis für natürliche Prozesse herauszubilden " (BUND 2002, 8). Aspekte einer Bildung für nachhaltige Entwicklung werden zunehmend in die Wildnisbildungsansätze der Nationalparke integriert (Langenhorst 2014; Langenhorst et al. 2014).

Grundsätzlich ist zu resümieren, dass die bisherige Debatte um Wildnisbildung aus der Perspektive der Nationalparke geführt wird, dem ein vorwiegend erfahrungsbasiertes Wissen zugrunde liegt. Trotz allem zeigen die Initiativen der Bundesregierung, der Stiftungen und Naturschutzverbände sowie die häufige Medienpräsenz des Themas Wildnis, dass die Problematik eine Alltagsrelevanz und Lebensbedeutsamkeit aufweist. So stellen Langenhorst und Lude (2014) in Anlehnung an eine Bildung für nachhaltige Entwicklung sieben Aspekte von Wildnisbildung zusammen, die eine Verbindung zwischen diesen beiden Bildungskonzepten herstellen soll:

"Harmonisch-chaotische Natur erleben: Das intensive Erleben von verwildernder, eigensinniger Natur zwischen Harmonie und Chaos, um Naturvertrautheit und ein realistisches Naturverständnis entwickeln zu können.

Achtsame Bewilderung erspüren: Das leibliche Erspüren der eigenen empfindlichen und lebendigen Natur des Menschen durch elementare Naturerfahrungen.

Ökologische Wechselwirkungen erkunden: Das konkrete Erfahren der ökologischen Wechselwirkungen zwischen Mensch und Mitwelt als Grundlage kompetenter Urteilsbildung im Alltag.

Lebensstil und Lebenssinn reflektieren: Das eingehende Nachdenken über Sinnfragen mit dem Blick auf den eigenen Lebensstil und die Begrenzung eigener materieller Ansprüche.

Wilde biologische Vielfalt entdecken: Das Entdecken und Erkunden der wilden biologischen Vielfalt mit ihrer Bedeutung für den Menschen.

Mit Wildnis auseinandersetzen: Die individuelle Erfahrung und Auseinandersetzung mit dem kulturellen Konzept «Wildnis» als Ergänzung zur Zivilisation.

Freiheit in der Wildnis erfahren: Das inspirierende Erleben der Wildnis als Ort persönlicher Freiheit in einer demokratischen Gesellschaft" (79 f.).

Diese Grundsätze von Wildnisbildung weisen eine Schnittmenge zu den Prinzipien einer Bildung für nachhaltige Entwicklung auf. So sind die ökologischen sowie sozialen Dimensionen deutlich erkennbar. Gleichzeitig erfolgt eine Betonung des kritischen und reflexiven Umgangs des Verhältnisses von Mensch und Umwelt. Durch das Erleben von (sekundärer) Wildnis sowie der Sensibilisierung für das eigene Handelns im Raum stellt der Ansatz der Wildnisbildung Verbindungen zur Forderung nach Partizipation, Vernetzung sowie dem Maßstabswechsel zwischen lokalem Handeln und globalem Denken dar. Zunehmend verstärkt sich die Relevanz der ökonomischen Dimension mit Blick auf das eigene Handeln anhand des persönlich geführten Lebensstils. Nicht zuletzt beinhaltet die Auseinandersetzung mit biologischen und geographischen Fachinhalten des Ökosystems Wald sowie mit Landnutzungsstrategien Fragestellungen der Nachhaltigkeit. Dadurch verspricht das Konzept als neuen Ansatzes einer Bildung für nachhaltige Entwicklung geeignet zu sein, da es die Dimensionen der Ökologie, Ökonomie und Soziales vereint. Insbesondere der Schwerpunkt der Handlungskompetenz mit dem persönlichen Eingebundensein in die Wildnis in Form von Camps erweitert eine Bildung für nach- 
haltige Entwicklung neben einer theoretischen auch um eine praktische Ebene. In Zukunft sollte hier der Zusammenhang zwischen Bildung für nachhaltige Entwicklung und Wildnisbildung noch stärker theoretisch aufgearbeitet und geprüft werden.

\subsection{Wildnisbildung für die universitäre Geogra- phielehrer/innenbildung und das Schulfach Geographie - ein neuer Ansatz?}

Das Unterrichtsfach Geographie ist aufgrund seiner Ziele, Inhalte und Funktionen dem Leitbild der Nachhaltigkeit und somit einer Bildung für nachhaltige Entwicklung besonders verpflichtet (Hemmer et al. 2013). Ein Leitziel des Schulfaches Geographie besteht in der Entwicklung eines umfassenden Verständnisses der Mensch-Umwelt-Beziehungen als hochkomplexes dynamisches System mit physischgeographischen und humangeographischen Subsystemen sowie deren Wechselwirkungen (DGfG 2014).

Um die Realisierung der Ziele im schulischen Umfeld gewährleisten zu können, kommt der universitären Fachlehrer/innenbildung eine wesentliche Bedeutung zu. Die von der Deutschen Gesellschaft für Geographie herausgegebenen Empfehlungen für die Lehrer/innenbildung im Fach Geographie beinhalten fachliche, fachdidaktische und bildungswissenschaftliche Kompetenzformulierungen, die der Befähigung zukünftiger Lehrkräfte dienen, um kompetenzorientierten Geographieunterricht planen, durchführen und reflektieren zu können. Die Bedeutsamkeit einer Bildung für nachhaltige Entwicklung innerhalb der Geographielehrer/ innenbildung wird dabei explizit hervorgehoben und stellt innerhalb der formulierten Kompetenzen eine wichtige Rahmung dar (DGfG 2010).

Um die Forderung einer Bildung für nachhaltige Entwicklung nicht nur als Thema, sondern als gelebtes und ganzheitliches Konzept in der Lehrer/innenbildung für Geographie sowie im Schulfach Geographie zu erfüllen, stellt sich die Frage, ob und inwiefern der Ansatz der Wildnisbildung dafür möglich ist. Literatur und Praxiserfahrungen lassen vermuten, dass Wildnisbildung für beides geeignet scheint, da Aspekte einer Bildung für nachhaltige Entwicklung thematisiert und erlebbar gemacht werden können (Langenhorst \& Lude 2014).

Das Konzept der Wildnisbildung ermöglicht Schülerinnen und Schülern und Lehramtsstudierenden den Aufenthalt in Schutzgebieten, die in der Regel nicht für einen mehrtägigen Aufenthalt freigegeben sind. Die Besonderheit und Exklusivität des Nationalparkbesuchs sowie des für Bildungszwecke vorgesehenen und infrastrukturell unterstützten Wildniscamps trägt vermutlich allein schon zu einer Sensibilisierung der Teilnehmenden für die Thematik Wildnis und
Beeinflussung des Schutzgebietes durch das eigene Handeln bei. Die Potenziale der Wildnisbildung als einen Ansatz für den Geographieunterricht sowie die universitäre Lehrer/innenbildung werden in der direkten Wahrnehmung des eigenen Handelns im Raum gesehen. Mit der Frage „Welche Spuren werden im Wildniscamp hinterlassen?" wird der Eingriff des Menschen am Beispiel eines gut abgrenzbaren Gebietes, in dem der Mensch nur Gast ist, sichtbar. Durch den Transfer auf das eigene Leben verändert sich die Perspektive und das Bewusstsein für die Integration in komplexe Systeme wird geschärft. Weiterhin rückt bei der Wahl des Lernortes Wildniscamp gewollt der Verzicht auf Komfort und das Prinzip der Bescheidenheit in den Fokus, um auch an dieser Stelle den Transfer zum eigenen Lebensstandard zu ermöglichen.

Aus Sicht der Lehrer/innenbildung umfasst ein erweitertes Konzept der Wildnisbildung die Professionalisierung von zukünftigen Lehrkräften, da der Ansatz neben dem fachlichen, handlungsorientierten, sozialen, konativen und reflexiven Diskurs zu Wildnis und Wildnisentwicklung sowie die eigene Rolle darin, um den Bereich der fachdidaktischen und pädagogischen Dimension erweitert wird. Die Lehramtsstudierenden sammeln neben dem eigenen Erleben von Wildnisbildung erste Erfahrungen im Konzipieren, Durchführen und Reflektieren von Lerneinheiten zur Wildnisbildung (Lindau 2015).

Bisher fehlen insgesamt abgesicherte Belege für die Sinnhaftigkeit und Wirksamkeit des Ansatzes der Wildnisbildung im Rahmen des Geographieunterrichts sowie in der Geographielehrer/innenbildung. Nachdem im Rahmen des Projektes „Wildnis macht stark" ein Konzept für die Lehrer/innenbildung entwickelt wurde, dass seit vier Jahren an der MartinLuther-Universität Halle-Wittenberg erfolgreich praktiziert wird, liegen nun erste Forschungsarbeiten vor. Diese gehen hauptsächlich der Frage nach der Wirksamkeit von Wildnisbildung in der Lehrer/innenbildung nach, von denen zwei Forschungsansätze im Kapitel 3 vorgestellt werden.

\section{Forschungsansätze zur Professionali- sierung von Lehramtsstudierenden am Beispiel Wildnisbildung}

\subsection{Forschungsziele und methodisches Vorgehen}

Im Rahmen des Projekts „Wildnis macht stark“ (gefördert durch die DBU, Laufzeit: 2012-2014) wurde eine Konzeption für ein Modul zur Wildnisbildung für Geographie-Lehramtsstudierende entwickelt und in der Praxis mehrfach erprobt. Ziel ist es, einen neuen Ansatz für die universitäre Lehrer/innenbildung zu 
schaffen, der die Entwicklung fachwissenschaftlicher, fachdidaktischer und pädagogischer Kompetenzen und Wissensbestände in sich vereint und gleichzeitig einen Ansatz für eine Bildung für nachhaltige Entwicklung bietet.

Ein wesentlicher Bestandteil der Wildnisbildungskonzeption ist der mehrmalige Aufenthalt in einem Wildniscamp im Nationalpark Harz. Die Aufenthalte dienen dazu, dass angehende Lehrer/innen des Faches Geographie die Idee der Wildnisbildung kennenlernen, gestalten und reflektieren, um sich als zukünftige Lehrkräfte zu professionalisieren. Bei dem Wildniscamp handelt es sich um einen durch minimale infrastrukturelle Maßnahmen unterstützten Lehr- und Lernort (Birkental im Westharz).

Ziel ist es, durch den emotionalen und authentischen Zugang durch den Ansatz der Wildnisbildung, einen Beitrag zur Professionalisierung von angehenden Lehrkräften für das Fach Geographie zu leisten. Durch die Kombination von fachwissenschaftlichen, fachdidaktischen sowie pädagogischen Inhalten, das Erleben der eigenen Person und der Gruppe in einer ungewohnten und für die meisten neuen Lernumgebung in Form eines Wildniscamps lassen sich Effekte hinsichtlich der Entwicklung der eigenen Persönlichkeit sowie des Professionswissens im Rahmen des Modells der professionellen Handlungskompetenz nach Baumert \& Kunter (2006) erwarten.

In mehreren Forschungsarbeiten wurde der Hauptfrage nachgegangen, inwieweit der Ansatz der Wildnisbildung zur Lehrer/innenprofessionalisierung während der universitären Phase im Rahmen des Geographiestudiums beitragen kann. Im weiteren Verlauf des Artikels werden zwei Forschungsarbeiten in wesentlichen Grundzügen vorgestellt.

\subsubsection{Forschungsansatz: Professionalisierung von Lehramtsstudierenden am Beispiel Wildnis- bildung - Eine explorative Längsschnittstu- die}

\section{Ziel und Fragestellung}

Das projektbegleitende Promotionsvorhaben geht der Frage nach, inwieweit Wildnisbildung zur Professionalisierung von zukünftigen Lehrkräften im Fach Geographie beitragen kann. Im Sinne einer professionalisierten Lehrer/innenbildung ist es notwendig, Lehramtsstudierende ganzheitlich zu befähigen, um diese Anforderungen zu erfüllen. Da Wildnisbildung als Möglichkeit einer Bildung für nachhaltige Entwicklung aufgefasst wird (Langenhorst et al. 2014; Hottenroth 2015; Lindau 2015), stellt sich die Frage, über welche Fähigkeiten und Fertigkeiten Lehramtsstudierende für Geographie verfügen sollten, um den erklärten Bildungsauftrag zu erfüllen.

\section{Methodisches Vorgehen}

Um die Forschungsfragen zu beantworten, wurden in einer explorativen Längsschnittstudie Interviewaussagen von Expertinnen und Experten für Wildnisbildung ( $\mathrm{n}=11)$ aus Deutschland (u.a. Nationalpark Harz, Bayrischer Wald, Martin-Luther-Universität Halle-Wittenberg) und den USA (u. a. Wilderness Institute, University of Montana) sowie von 17 Lehramtsstudierenden des Faches Geographie (Gruppe 1: $\mathrm{n}=9$; Gruppe 2: $\mathrm{n}=8$ ), als Teilnehmende am Projekt "Wildnis macht stark“, gegenübergestellt. Die Studierenden wurden vor und jeweils nach der Teilnahme an den einzelnen Teilmodulen und Wildniscamps (s. Kap. 3.2) befragt. Die Daten wurden aus insgesamt 64 Interviews erhoben und mithilfe der qualitativen Inhaltsanalyse nach Mayring (2015) und Gropengießer (2008) ausgewertet. Dabei werden die Aussagen der Expertinnen und Experten mit denen der Lehramtsstudierenden verglichen, um so ein Bild über die leitenden und teilnehmenden Akteure der Wildnisbildung zu erhalten. Die Ergebnisse hinsichtlich der Einschätzung von Zielen und Merkmalen von Wildnisbildung sowie deren Eignung für die universitäre Lehrer/innenbildung werden zu einem Konsens zusammengeführt, um schließlich eine Empfehlung für die Implementierung von Wildnisbildung als einen Ansatz zur Lehrer/innenprofessionalisierung im Bereich Bildung für nachhaltige Entwicklung für den Geographieunterricht zu entwickeln. Um Potenziale und Grenzen von Wildnisbildung für die Lehrer/innenprofessionalisierung herauszustellen, ergänzt das Vorhaben eine SWOT-Analyse (Stärken-SchwächenChancen-Risiken-Analyse) für das Bildungskonzept. Mittels dieser Positionierungsanalyse wird dem gegenwärtigen Stärken-Schwächen-Profil aus der praktischen Umsetzungsphase des Projektes „Wildnis macht stark" sowie aus den Erfahrungen von Expertinnen und Experten ein Chancen-Risiko-Profil zur Durchführung von Wildnisbildung gegenüber gestellt, welches zur Etablierung dieses Bildungskonzeptes in der Lehrer/innenbildung dienen soll.

\subsubsection{Forschungsansatz: Einstellungsänderungen von Lehramtsstudierenden durch Wildnis- camps}

\section{Ziel und Fragestellung}

Im Rahmen der Begleitforschung durch die MartinLuther-Universität Halle-Wittenberg wurde erhoben, ob sich die Einstellungen der Teilnehmenden am Modul mit dem Schwerpunkt des Aufenthalts im Wildniscamp ändern. Hierfür fand ein Instrument Einsatz, das in der Fachliteratur als CBAM (Concern Based Adoption Model) bekannt ist. Unter Concern versteht Fuller (1969) die Beschreibung der eigenen Wahrneh- 
Tab. 1: Stufen der Zustimmung zu einer Innovation, die typischerweise durchlaufen werden (nach Hall \& Hord 2006).

\begin{tabular}{ll}
\hline Stage/Stufe & Typisches Verhalten \\
\hline O Bewusstsein & die/der Befragte kennt die Neuerung oder er/sie kennt sie nicht \\
1 Information & die/der Befragte möchte mehr über die Neuerung wissen \\
2 Persönliche Betroffenheit & die/der Befragte reflektiert die eigene Einstellung \\
3 Aufgabenmanagement & die/der Befragte fragt sich, ob sie/er den Anforderungen gewachsen ist \\
4 Auswirkung auf Lernende & die/der Befragte fragt nach Verbesserungen für die Schüler/innen \\
5 Kooperationsmöglichkeiten & $\begin{array}{l}\text { die/der Befragte sucht nach Partner/innen für die Umsetzung } \\
\text { 6 Revision und Optimierung }\end{array}$ \\
& $\begin{array}{l}\text { die/der Befragte hat so viele Erfahrungen, dass sie/er am Konzept weiterarbeiten } \\
\text { möchte }\end{array}$ \\
\hline
\end{tabular}

mungen und Emotionen im Hinblick auf verschiedene Aspekte der eigenen (pädagogischen) Arbeit (Pant et al. 2008). Das Instrument wurde vor rund 30 Jahren für die Beobachtung von Novizinnen und Novizen im Lehramt entwickelt und dient seither zur Beschreibung von Einstellungsänderungen bei erlebten Neuerungen (Hall \& Hord 2006). Das dahinter liegende Modell geht davon aus, dass idealtypisch jede Novizin/jeder Novize sieben Stufen der Akzeptanz einer Neuerung (Innovation) durchläuft. Diese Stufen werden in Tabelle 1 dargestellt.

Bezogen auf die Implementierung von Wildnisbildung innerhalb der universitären Lehrer/innenbildung für Geographie stellt sich die Frage, inwieweit sich die Einstellungen von Lehramtsstudierenden gegenüber dem Lernort Wildniscamp verändern. Da den meisten Studierenden die Form des Wildniscamps nicht bekannt ist und durch die persönliche Eingebundenheit innerhalb des Lernsettings eine hohe Affektivität erwartet werden kann, ist die Frage nach der Akzeptanz von Wildniscamps als Lernort bedeutsam.

\section{Methodisches Vorgehen}

Zur Ermittlung des jeweiligen Einstellungsprofils gegenüber Wildniscamps wurde der Stages-of-ConcernFragebogen (Ebenda 2006) eingesetzt. Innerhalb des Fragebogens werden zu der siebenstufigen Skala der
Akzeptanz von Neuerungen - in diesem Fall von Wildniscamps als Lernort - Zustimmung oder Ablehnung angekreuzt. Die Concerns beziehen sich einerseits auf die Situation der Befragten, andererseits auf die Perspektive der zukünftigen Zielgruppe der zu unterrichtenden Schüler/innen und schließlich auf die Zusammenarbeit zwischen zukünftigen Lehrkräften und Schüler/innen. Für jede dieser Akzeptanzstufen (s. Tab. 1) liegen 5 typische Aussagen vor, die aus dem Stages-of-Concern-Fragebogen übernommen und auf Wildniscamps angepasst wurden. Die Aussagen sind in der Reihenfolge gemischt, die einzelnen Akzeptanzstufen den Befragten nicht bekannt. Tabelle 2 zeigt beispielhaft einige Items aus dem Fragebogen.

Die Teilnehmer/innen der Wildniscamps wurden vor und nach dem Aufenthalt im Wildniscamp des Nationalparks Harz befragt.

\subsection{Darstellung der Intervention}

Im Folgenden soll der Ablauf des Moduls „Wildnisbildung " als Bestandteil der universitären Lehrer/innenbildung für das Unterrichtsfach Geographie näher beschrieben werden, da diese gleichzeitig die Intervention innerhalb der bereits vorgestellten Forschungsansätze und die Grundlage für die Datenerhebung darstellt (Tab. 3).

Tab. 2: Ausschnitt aus dem Stages-of-Concern-Fragebogen. 1-7: Zunehmende Zustimmung. 0: keine Aussage möglich. Kursiv in der letzten Spalte: Stufe des Concerns (s. Tab. 1, wird im Fragebogen nicht angegeben).

\begin{tabular}{|c|c|c|c|c|c|c|c|c|c|}
\hline & 1 & 2 & 3 & 4 & 5 & 6 & 7 & 0 & \\
\hline $\begin{array}{l}\text { Es interessiert mich, was die Schüler/innen oder die Jugendlichen vom Wildniscamp } \\
\text { halten werden. }\end{array}$ & 0 & 0 & 0 & 0 & o & 0 & 0 & 0 & 4 \\
\hline $\begin{array}{l}\text { Ich beschäftige mich gerade mit anderen Neuerungen für die Umweltbildung und } \\
\text { nicht mit dem Wildniscamp. }\end{array}$ & 0 & 0 & 0 & 0 & o & o & 0 & 0 & 0 \\
\hline $\begin{array}{l}\text { Ich fürchte, ich habe gar nicht genügend Vorbereitungszeit, um immer wieder Wild- } \\
\text { niscamps durchführen zu können. }\end{array}$ & 0 & 0 & 0 & 0 & o & o & 0 & o & 2 \\
\hline $\begin{array}{l}\text { Ich bin gerne bereit, anderen Kollegen/innen oder Kommilitonen/innen bei der Ent- } \\
\text { deckung des Wildniscamps zu unterstützen. }\end{array}$ & ० & 0 & 0 & 0 & o & o & o & o & 5 \\
\hline
\end{tabular}


Tab. 3: Kurzbeschreibung der Intervention im Rahmen des Moduls „Wildnisbildung“ für Lehramtsstudierende im Fach Geographie.

\begin{tabular}{|c|c|c|}
\hline Modulname & Ziele & Veranstaltungen \\
\hline \multicolumn{3}{|c|}{ Modul im Rahmen des Lehramtsstudiums (5 Leistungspunkte) } \\
\hline $\begin{array}{l}\text { Teilmodul 1: } \\
\text { Wildnis erleben }\end{array}$ & $\begin{array}{l}\text { Die Lehramtsstudierenden sind in der } \\
\text { Lage, die Aspekte der Neuentwicklung } \\
\text { von (Sekundär-) Wildnis exemplarisch } \\
\text { im Nationalpark Harz zu erleben und } \\
\text { zu reflektieren. Sie können die Entwick- } \\
\text { lung der Wildnisbildung in Deutsch- } \\
\text { land beschreiben. }\end{array}$ & $\begin{array}{l}\text { Vorbereitungsseminar ( } 4 \text { Std. im Sommersemester } \\
\text { des 1. Jahres) } \\
\text { Wildniscamp } 1 \mathrm{im} \text { Birkental bei Lonau ( } 3 \text { Tage im } \\
\text { September des 1. Jahres) } \\
\text { Nachbereitungsseminar (4 Std. im Wintersemester } \\
\text { des 1. Jahres) }\end{array}$ \\
\hline $\begin{array}{l}\text { Teilmodul 2: } \\
\text { Wildnisbildung begrei- } \\
\text { fen }\end{array}$ & $\begin{array}{l}\text { Die Lehramtsstudierenden sind in der } \\
\text { Lage, Wildnisbildung zu begreifen bzw. } \\
\text { den Perspektivwechsel von Teilneh- } \\
\text { menden (Lernenden) zu Teamerinnen } \\
\text { und Teamern (Lernbegleiterinnen und } \\
\text { Lernbegleitern) von Wildnisbildung zu } \\
\text { vollziehen. }\end{array}$ & $\begin{array}{l}\text { Vorbereitungsseminar ( } 12 \text { Std., im Sommersemes- } \\
\text { ter des } 2 \text {. Jahres) } \\
\text { Wildniscamp } 2 \text { im Birkental bei Lonau ( } 5 \text { Tage im } \\
\text { Juli / August des } 2 \text {. Jahres) } \\
\text { Nachbereitungsseminar ( } 4 \text { Std. im Wintersemester } \\
\text { des 2. Jahres) }\end{array}$ \\
\hline \multicolumn{3}{|c|}{ Zertifikat für Multiplikatorinnen und Multiplikatoren } \\
\hline $\begin{array}{l}\text { Teilmodul 3: } \\
\text { Wildnisbildung gestal- } \\
\text { ten }\end{array}$ & $\begin{array}{l}\text { Die Studierenden sind in der Lage, als } \\
\text { Multiplikatorinnen und Multiplikatoren } \\
\text { Lerneinheiten zur Wildnisbildung zu } \\
\text { gestalten. }\end{array}$ & $\begin{array}{l}\text { Vorbereitungsseminar (4 Std. im Sommer des } 2 . \\
\text { Jahres, in der Regel nach Teilmodul 2) } \\
\text { Wildniscamp } 1 \mathrm{im} \text { Birkental bei Lonau ( } 3 \text { Tage im } \\
\text { September des 2. Jahres) } \\
\text { Nachbereitungsseminar (4 Std. im Wintersemester } \\
\text { des 2. Jahres) }\end{array}$ \\
\hline $\begin{array}{l}\text { Teilmodul 4: } \\
\text { Wildnisbildung vor der } \\
\text { Haustür }\end{array}$ & $\begin{array}{l}\text { Die Studierenden sind in der Lage, } \\
\text { Ideen zur Umsetzung von Wildnisbil- } \\
\text { dung im Heimatraum zu entwickeln, } \\
\text { durchzuführen und zu reflektieren. }\end{array}$ & $\begin{array}{l}\text { Vorbereitungsseminar (4 Std. im Wintersemester } \\
\text { des 2. Jahres) } \\
\text { Exkursion im Stadtgebiet von Halle/Saale (4 Std. } \\
\text { im Dezember des 2. Jahres) } \\
\text { Nachbereitungsseminar ( } 2 \text { Std. im Dezember des } \\
\text { 2. Jahres) }\end{array}$ \\
\hline
\end{tabular}

Die Teilnahme an der Multiplikator/innenqualifizierung „Wildnis macht stark“ ist in das Lehramtsstudium im Fach Geographie im Bereich Fachdidaktik als Wahlpflichtmodul integriert. Die Multiplikator/ innenqualifizierung ist zweistufig (Dauer: $1 \mathrm{Jahr}$ ) oder vierstufig (Dauer: 1,5 Jahre) aufgebaut. Beim zweistufigen Modul werden 5 Leistungspunkte, die für das Geographiestudium angerechnet werden können, erworben. Das vierstufige Modell wird mit einem Zertifikat „Multiplikator/in für Wildnisbildung“ des Nationalparks Harz abgeschlossen.

Im folgenden Abschnitt werden die inhaltliche und organisatorische Umsetzung der Teilmodule der Intervention ausführlicher beschrieben.

\section{Teilmodul 1: Wildnis erleben}

Im Vorbereitungsseminar erhalten die Lehramtsstudierenden durch eine Nationalparvertreterin/einen Nationalparkvertreter und eine Univeristätslehrende/ einen Universitätslehrenden einen ersten Einblick in das Konzept, in dem Literaturstudien und kritische Seminardiskussionen zu den Begriffen „Wildnis“ und
„Wildnisbildung“ sowie über das Konzept des Nationalparks Harz erfolgen. Im Vordergrund steht die reflexive Annäherung an den in Deutschland teilweise kontrovers diskutierten Ansatz.

Das Wildniscamp findet im Herbst statt. Der drei Kilometer lange Weg zum Camp wird durch die Studierenden mithilfe von Karte und Kompass ermittelt.

Nach der Ankunft im Camp werden die Schlafstätten und der Gemeinschaftsplatz mithilfe von Planen bzw. Sheltern errichtet. Zentraler Ort des Gemeinschaftsplatzes ist die Feuerstelle, die auch zum Kochen und besonders am Abend oder während Schlechtwetterphasen als Versammlungs- und Gesprächsort dient. Die Toiletten und ein Dusch- bzw. Waschplatz ergänzen die Campausstattung. Neben der Sicherung der Grundbedürfnisse (trockener und warmer Schlafund Gemeinschaftsplatz, Feuer, Ernährung), die einen großen Teil der Zeit einnehmen, wird der Aufenthalt im Camp für die kritische Auseinandersetzung mit den Themenbereichen Wildnis und Wildnisbildung genutzt. Dazu gehören Beobachtungen und kleinere Wanderungen innerhalb des Nationalparks, in denen 


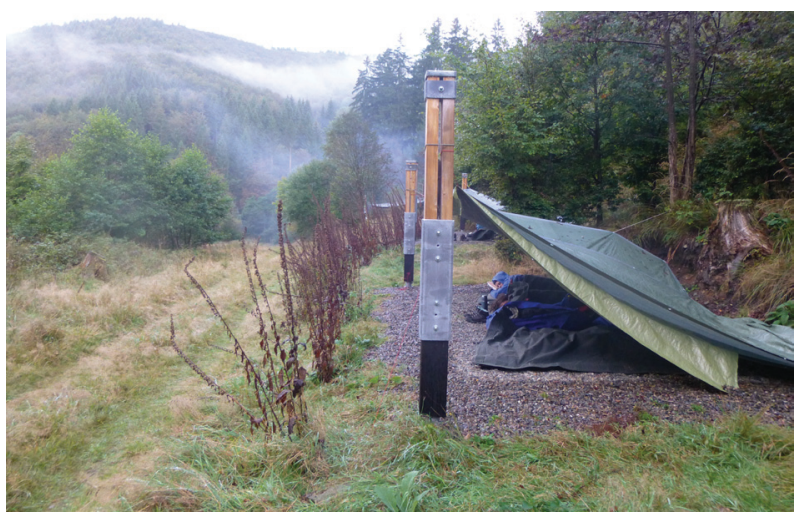

Abb.1: Schlafstelle als infrastrukturelle Einrichtung im Wildniscamp Birkental (Harz).

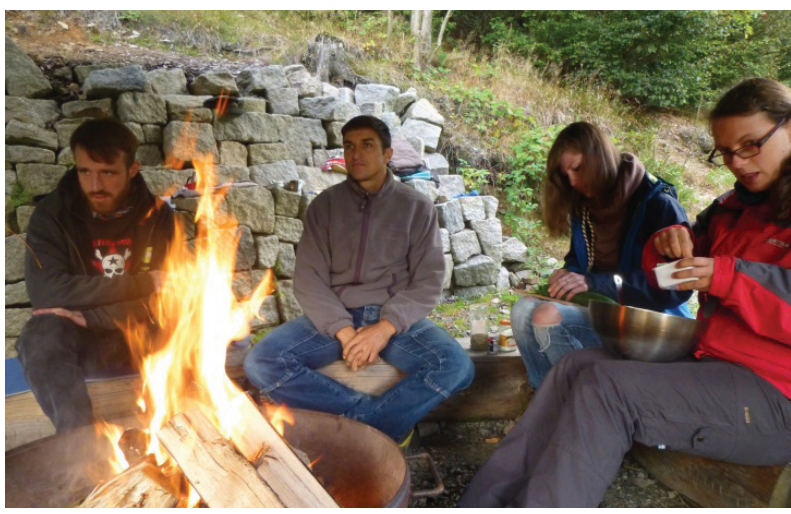

Abb. 2: Gemeinsame Essensvorbereitung am Feuer des Gemeinschafts- und Versammlungsplatzes.

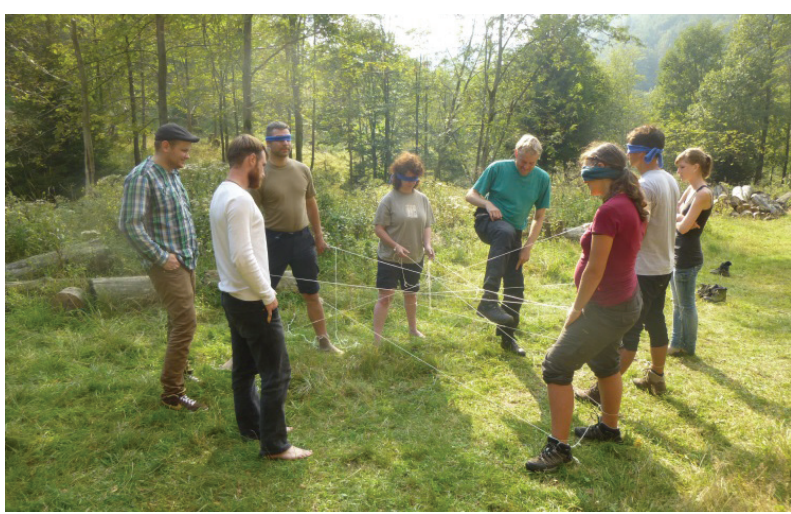

Abb. 3: Teilnehmende bei einem Wahrnehmungsspiel zur Sensibilisierung der Sinne als Bestandteil der Wildnisbildung.

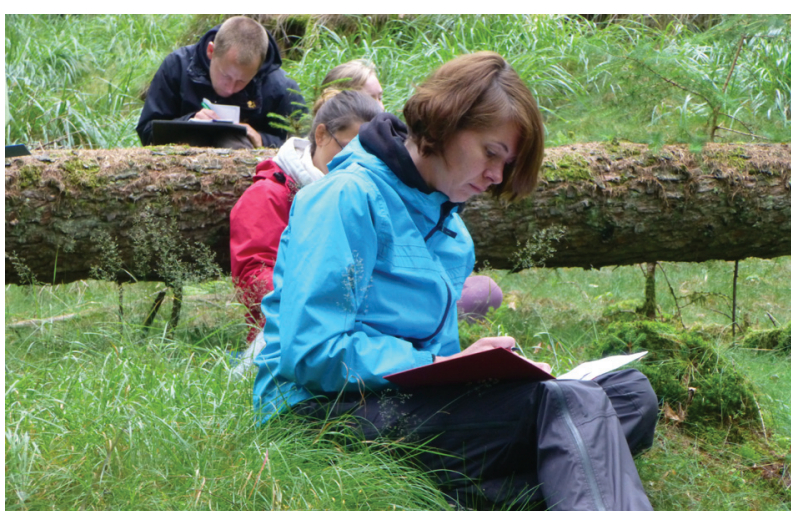

Abb. 4: Portfolio-Arbeit. besonders der Prozessschutz sowie das „Natur Natur sein lassen" (Bibelriehter 1998) thematisiert werden. In Diskussionsrunden wird außerdem der eigene Lebensstil vor dem Hintergrund einer Bildung für nachhaltige Entwicklung reflektiert. Für die persönlichen Reflexionen werden Portfolios genutzt. Übungen, die aus dem Coyote-Coaching stammen, unterstützen die Sensibilisierung für Wildnis und Wildnisbildung. Dazu gehören neben einem täglichen Sit Spot (englisch für Sitzplatz), Wahrnehmungsübungen, die Kunst des Fragenstellens, Bestimmungsübung, Schleichen, Suchen und Spielen. Ebenso gehören der Campaufbau, das Feuermachen, das Wasserfiltern und das Kochen zur Erstbegegnung mit dem Konzept (Abb. 1-4).

Das erste Modul wird nach Beendigung des Wildniscamps in einem weiteren Seminar reflektiert, indem über die Eindrücke zur Wildnis, Wildnisbildung und zum Wildniscamp diskutiert wird. Das Modul legt den Schwerpunkt innerhalb des Modells der professionellen Handlungskompetenz (Baumert \& Kunter 2006) auf die Wissensdimension des „Fachwissens“ sowie auf die affektive Erlebenskomponente.

\section{Teilmodul 2: Wildnisbildung begreifen}

Während der Vorbereitungsseminare für das Wildniscamp 2 wird der Perspektivwechsel von der/von dem Teilnehmenden zum/zur Teamer/in vorbereitet, indem den Studierenden mehr Verantwortung zur Realisierung des Wildniscamps übertragen wird. Auf der Grundlage der Bildungsstandards für die Lehrer/ innenbildung (KMK 2004; KMK 2013) sowie der Bildungsstandards für den Mittleren Schulabschluss im Fach Geographie (DGfG 2014) entwickeln die Studierenden Lerneinheiten, die innerhalb der Gruppe durchgeführt und reflektiert werden. Thematische Ideen bzw. geeignete Methoden gewinnen sie aus der Literatur zur Wildnisbildung (z. B. Coyote-Teaching: Young et al. 2014), die auf die Fragestellungen von Schule und Geographieunterricht angepasst werden. Diese Überlegungen werden allen Teilnehmenden zur Verfügung gestellt, wodurch ein Fundus an Wildnisbildungseinheiten im Portfolio angelegt wird, der auch für den schulischen Einsatz geeignet ist. Ziel ist es, besonders die Dimensionen „Fachdidaktisches Wissen“ und „Pädagogisches Wissen“ auf der Grundlage eines soliden Fachwissens zur Wildnisdiskussion in Deutschland im Rahmen des Professionswissens von Lehramtsstudierenden in realen Lernsituationen weiterzuentwickeln. Da die Örtlichkeiten und Gegebenheiten des Wildniscamps aus dem ersten Aufenthalt bekannt sind, liegt die Organisation des Campaufbaus zunehmend in den Händen der Studierenden. Die Verantwortung für das Camp wechselt täglich. Während des Teilmoduls 2 wird Wildnisbildung mithilfe von Beobachtungen, Wahrnehmungs- und Reflexi- 
onsübungen erörtert. Neben Diskussionen zu Potenzialen und Grenzen von Wildnis, Wildnisbildung und geeigneten Örtlichkeiten außerhalb des Nationalparks nehmen Aspekte zur Organisation von Wildniscamps für Schüler/innen zunehmend Raum ein. Dadurch werden die Voraussetzungen, die eine Teamerin/ein Teamer benötigt, zunehmend in den Fokus der zukünftigen Lehrkräfte gerückt und ein Beitrag zur Lehrer/innenprofessionalisierung in allen Dimensionen, besonders im Bereich des pädagogischen Wissens, geleistet. Reflexionszeiten allein und in der Gruppe ergänzen den Aufenthalt im Wildniscamp. Dazu gehört der Sit Spot, aber auch die Portfolio-Arbeit. Ergänzt wird eine freie Schwerpunktsetzung der Reflexion durch konkrete Aufgaben, in dem die Studierenden die Entwicklung des eigenen Professionswissens beurteilen.

Nach erfolgreicher Absolvierung der ersten beiden Teilmodule ist das Wahlpflichtmodul abgeschlossen. Die Studierenden haben darüber hinaus die Möglichkeit, ein Zertifikat als Multiplikator/in für Wildnisbildung durch den Besuch der Teilmodule 3 und $4 \mathrm{zu}$ erwerben.

\section{Teilmodul 3: Wildnisbildung gestalten}

Im Teilmodul 3 sind die Studierenden sehr stark in die Planung, Durchführung und Reflexion des Teilmoduls 1 in Kooperation mit dem Nationalpark Harz eingebunden. Zwei bis drei Studierende können als angehende Multiplikatorinnen und Multiplikatoren das Teilmodul 1 des nachfolgenden Jahrgangs begleiten. Die Multiplikatorinnen und Multiplikatoren sind u. a. für die Camp-Organisation verantwortlich. Sie leiten selbständig Einheiten zur Wildnisbildung an und unterstützen die Teamer/innen des Nationalparks Harz und der Universität.

\section{Teilmodul 4: Wildnisbildung vor der Haustür}

Das abschließende Teilmodul 4 hat zum Ziel, Wildnisbildung in den Heimatraum der Teilnehmenden zu transferieren. Der urbane Raum Halle/Saale als Universitätsstandort weist Potenziale aber auch Grenzen für Wildnisbildung auf. Die Möglichkeiten und Einschränkungen von urbaner Wildnisbildung sowie geeigneten Lernorten werden in einem Vorbereitungsseminar erörtert, indem auch Methoden der Wildnisbildung an geeigneten Standorten in Halle vorbereitet und kritisch reflektiert werden. Das Teilmodul 4 findet im Dezember statt, um neben dem Wechsel der Örtlichkeit auch einen jahreszeitlichen Transfer zu realisieren. Dadurch sollen die Potenziale und Barrieren der Wildnisbildung in der kälteren und dunkleren Jahreszeit herausgearbeitet werden.

Zum Abschluss des Moduls werden am Lagerfeuer die Zertifikate in einem würdigen Rahmen an die Multiplikatorinnen und Multiplikatoren sowie Teilnahmebescheinigungen an die Teilnehmenden der ersten beiden Teilmodule überreicht.

\subsection{Ausgewählte Ergebnisse der Forschungsan- sätze}

\subsubsection{Forschungsansatz: Professionalisierung von Lehramtsstudierenden am Beispiel Wildnis- bildung - Eine explorative Längsschnittstu- die}

Die projektbegleitende Langzeitstudie ermittelte aus Sicht der Studierenden (Gruppe 1: $\mathrm{n}=9$ ) nach der Teilnahme am Wildnismodul (Post Teilmodul 2) folgende Ergebnisse (Tab. 4): insbesondere das fachdidaktische Wissen ist zur Umsetzung von Wildnisbildung bedeutsam, welches das Wissen und die

Tab. 4: Fähigkeiten und Fertigkeiten zur Umsetzung von Wildnisbildung aus Sicht der Studierenden nach Teilmodul 2, (Gruppe 1: $n=9)$ (Quelle: Hottenroth 2015).

\begin{tabular}{|c|c|c|c|}
\hline \multicolumn{4}{|c|}{ Post Teilmodul 2 „Wildnisbildung begreifen“ } \\
\hline Rang & Fähigkeiten und Fertigkeiten zur Umsetzung von Wildnisbildung & $\begin{array}{l}\text { Nennungen in \% } \\
\text { (Anzahl) }\end{array}$ & $\begin{array}{l}\text { Personen in \% } \\
\text { (Anzahl) }\end{array}$ \\
\hline 1 & Fachdidaktisches Wissen & $20 \%(20)$ & $82 \%(9)$ \\
\hline 2 & Erfahrung & $18 \%(18)$ & $67 \%(6)$ \\
\hline 2 & Organisationskompetenz & $11 \%(11)$ & $67 \%(6)$ \\
\hline 3 & Fachliches Wissen & $11 \%(11)$ & $67 \%(6)$ \\
\hline 4 & Pädagogisches Wissen & $11 \%(11)$ & $56 \%(5)$ \\
\hline 5 & Motivationskompetenz & $7 \%(7)$ & $18 \%(2)$ \\
\hline 6 & Authentizität & $3 \%(3)$ & $18 \%(2)$ \\
\hline 6 & Räumliche Orientierungskompetenz & $3 \%(3)$ & $18 \%(2)$ \\
\hline 6 & Sich-wohl-Fühlen & $2 \%(2)$ & $18 \%(2)$ \\
\hline
\end{tabular}


Aufbereitung fachdidaktischer Inhalte bzgl. Wildnisbildung beinhaltet. Sechs von neun Studierenden sehen die Erfahrung im Bereich Wildnisbildung sowie die Organisationskompetenz zur Planung, Durchführung und Reflexion von Wildnisbildungseinheiten als wichtige Fähigkeiten und Fertigkeiten an.

Tabelle 5 zeigt die zu entwickelnden Kompetenzen von angehenden Lehrerinnen und Lehrern im Bereich Wildnisbildung aus Sicht der befragten Expertinnen und Experten $(\mathrm{n}=11)$. Demnach sollten sie v. a. sozial kompetent sein, um verschiedene Gruppendynamiken, die z. B. durch das Zusammenleben im Wildniscamp entstehen, zu erkennen sowie situationsangepasst agieren zu können (9 von 9 Personen). Alle Expertinnen und Experten gaben an, dass Wildnisbildner/innen in ihrer Art und Weise authentisch sein sollten, d.h. ihre Einstellung gegenüber Wildnisbildung z. B. mit dem Ziel eines nachhaltigen Lebensstils auch vorzuleben. Acht von neun Expertinnen und Experten erklärten außerdem, dass pädagogisches Wissen eine Voraussetzung für angehende Wildnisbildner/innen sein sollte, welches Wissen über die allgemeinen Prinzipien der Gruppenorganisation und des Gruppenmanagements umfasst.

Erste Ergebnisse des Datenvergleichs zeigen, dass die Expertinnen und Experten sowie Lehramtsstudierenden ähnliche Fähigkeiten und Fertigkeiten zur Umsetzung von Wildnisbildung nennen, jedoch unterschiedlich gewichten. Einfluss nehmen hierbei die differenzierten Ausprägungen in den individuellen Kompetenzständen der erfahrenen Expertinnen und Experten im Vergleich zu Lehramtsstudierenden, die erst wenige Erfahrungen im Bereich der Wildnisbildung nach dem Forschungsprojekt aufweisen können.
Übereinstimmend bewerteten die Befragten Wildnisbildung als eine mögliche Facette einer Bildung für nachhalte Entwicklung mit dem Ziel, ein nachhaltiges Denken und Handeln zu fördern. Als Merkmal beschrieben beide Zielgruppen das Bescheidenheitsprinzip, welches v.a. durch das einfache Leben im Wildniscamp erfahrbar ist. Das Erleben von Bescheidenheit kann schließlich durch Reflexion zum Hinterfragen des eigenen (nicht-)nachhaltigen Lebensstils führen (Langenhorst \& Lude 2014).

Die Ergebnisse der SWOT-Analyse zeigen auf, dass Expertinnen und Experten und Lehramtsstudierende als vorrangige Stärke von Wildnisbildung das Ermöglichen von intensiven Erfahrungen auf Grund des besonderen Lehr- und Lernsettings v. a. im Nationalpark benennen. Der Grad der Wildheit dieses Lehr- und Lernortes ist dabei besonders für die Studierenden entscheidend, um Wildnisbildung authentisch erfahren zu können. Einigkeit besteht über die Chance von Wildnisbildung, zusätzlich alternative Räume außerhalb des Nationalparks zur Umsetzung des Bildungskonzeptes i. S. einer urbanen Wildnisbildung zu nutzen (z. B. wilde Ecke auf dem Schulhof, nahegelegener Stadtwald), um v. a. Schüler/innen in ihrer näheren Umgebung für das Konzept zu sensibilisieren. Hierbei kann der Fokus auf die Entstehung einer Sekundärwildnis auf ehemals bebauten Flächen gelegt werden.

Tab. 5: Fähigkeiten und Fertigkeiten zur Umsetzung von Wildnisbildung aus Sicht der Expertinnen und Experten, $(n=11)$ (Quelle: Hottenroth 2015).

\begin{tabular}{llrr}
\hline Rang & $\begin{array}{l}\text { Fähigkeiten und Fertigkeiten zur Umsetzung } \\
\text { von Wildnisbildung }\end{array}$ & $\begin{array}{l}\text { Nennungen in \% } \\
\text { (Anzahl) }\end{array}$ & $\begin{array}{l}\text { Personen in \% } \\
\text { (Anzahl) }\end{array}$ \\
\hline 1 & Sozialkompetenz & $11 \%(21)$ & $82 \%(9)$ \\
1 & Authentizität & $8, \%(16)$ & $82 \%(9)$ \\
2 & Pädagogisches Wissen & $9 \%(18)$ & $73 \%(8)$ \\
3 & Motivationskompetenz & $5 \%(10)$ & $64 \%(7)$ \\
4 & Erfahrung & $13 \%(26)$ & $55 \%(6)$ \\
4 & Fachdidaktisches Wissen & $12 \%(23)$ & $55 \%(6)$ \\
4 & Reflexionskompetenz & $6 \%(12)$ & $55 \%(6)$ \\
4 & Fachliches Wissen & $7 \%(11)$ & $55 \%(6)$ \\
5 & Risikomanagement-Fähigkeiten & $9 \%(17)$ & $55 \%(6)$ \\
5 & Organisationskompetenz & $8 \%(16)$ & $45 \%(5)$ \\
\hline
\end{tabular}

* Mehrfachnennungen in einem Interview möglich

"* Anzahl der Personen (Expertinnen und Experten), die sich zur genannten Kategorie geäußert haben 


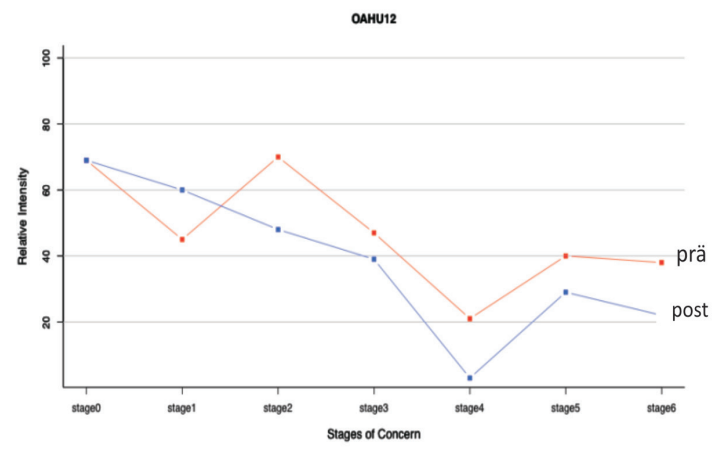

Abb. 5: Dielder nicht Überzengte bleibt skeptisch.

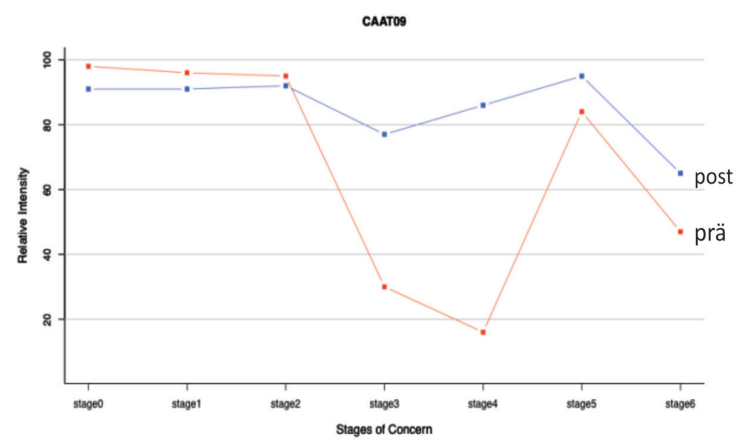

Abb. 7: Dielder anfangs Besorgte wird überzeugt.

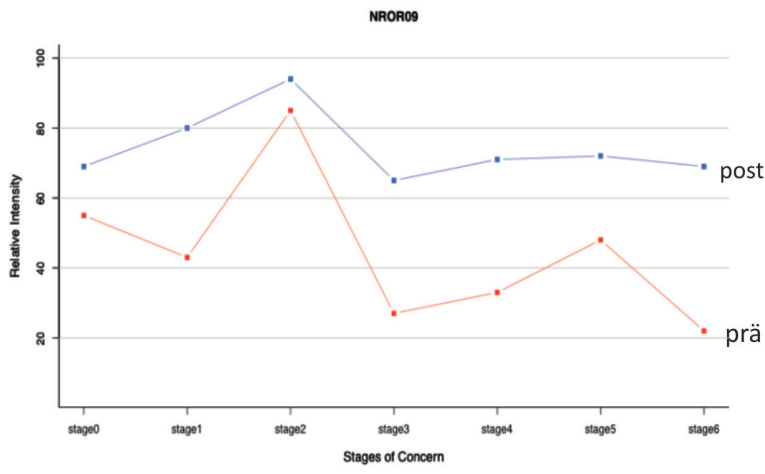

Abb. 6: Dielder vorher Skeptische wird überzeugt.

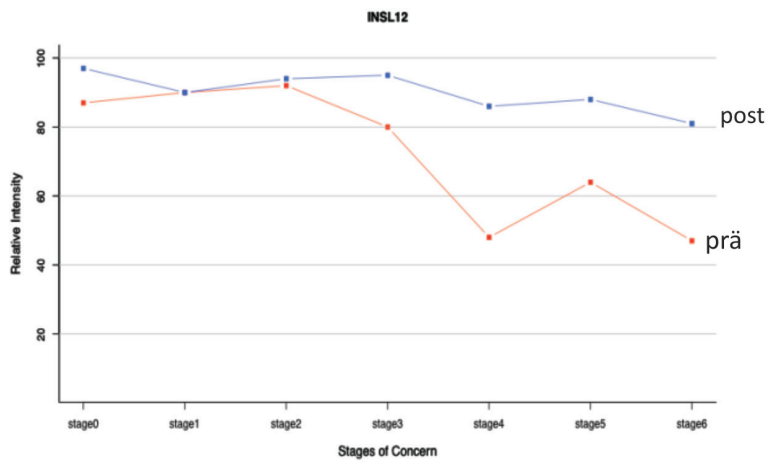

Abb. 8: Dielder insgesamt Bereite wird komplett überzeugt.

Abb. 5-8: Einstellungsmessung von Lehramtsstudierenden ( $n=70$ ) gegenüber Wildniscamps mithilfe der CBAM-Methode (nach Hall \& Hord 2006). Dargestellt sind vier Antwortmuster von vier verschiedenen Teilnehmer/innen zu den sieben Stufen der Akzeptanz von Wildniscamps (O Bewusstsein, 1 Information, 2 Persönliche Betroffenheit, 3 Aufgabenmanagement, 4 Auswirkungen auf Lernende, 5 Kooperationsmöglichkeiten, 6 Revision und Optimierung).

Tab. 6: Häufigkeit der Typen aus den Abb. 5-8 in der ersten Kohorte $(n=24)$.

\begin{tabular}{llr}
\hline Abbildung (s. o.) & Typ & Anzahl \\
\hline Abb. 5 & Die/der nicht Überzeugte bleibt skeptisch. & 2 \\
Abb. 6 & Die/der vorher Skeptische wird überzeugt. & 13 \\
Abb. 7 & Die/der anfangs Besorgte wird überzeugt. & 4 \\
Abb. 8 & Die/der insgesamt Bereite wird komplett überzeugt. & 5 \\
\hline
\end{tabular}

\subsubsection{Forschungsansatz: Einstellungsänderungen von Lehramtsstudierenden durch Wildnis- camps}

Den Ergebnissen der Befragung zu den Einstellungsänderungen von Lehramtsstudierenden durch den Besuch eines Wildniscamps lassen sich verschiedene Antwortmuster der Teilnehmenden zuordnen. Vier dieser Muster werden hier wiedergegeben (Abb. 5-8). Dabei sind die Prä-Kurven jeweils aus Daten ermittelt, die mithilfe eines Fragebogens vor dem Wildniscamp erhoben wurden, die Post-Kurven aus Daten am Ende des Camps. Die Punkte geben jeweils die Mitte aus den angekreuzten Werten in den fünf verschiedenen
Items zu den jeweiligen Stufen (Stages) an. Sie wurden in den Grafiken zur besseren Vergleichbarkeit der Befragungszeitpunkte durch Linien verbunden.

Die dargestellten Antwortmuster zeigen generell eine hohe Akzeptanz gegenüber Wildniscamps als Lehr- und Lernort zur Umsetzung einer Bildung für nachhaltige Entwicklung im Rahmen der universitären Lehrer/innenbildung. Dies zeigt sich sowohl am Antwortmuster der/des vorher Skeptischen, der durch die Intervention in Wildniscamps überzeugt wurde, als auch bei der/dem anfangs Besorgten und dann Überzeugten sowie der/dem schon vorher als auch nachher komplett Überzeugten (Abb. 6-8). Einzelne Antwortmuster zeigen jedoch, dass keine flächende- 
ckende Akzeptanz des Wildniscamp als Bildungsinnovation festgestellt wurde (Abb. 5). Eine Auswertung der Häufigkeit der Antwortmuster wurde nach den ersten 24 Befragungen durchgeführt. Sie ergab die wie in Tabelle 6 dargestellte Verteilung.

\section{Diskussion und Ausblick}

Das Konzept sowie die Umsetzung des Moduls im Rahmen der Lehrer/innenbildung im Unterrichtsfach Geographie an der Martin-Luther-Universität HalleWittenberg beschreitet einen neuen Weg innerhalb der Lehrer/innenprofessionalisierung als Teil einer Bildung für nachhaltige Entwicklung. Durch einen zeitlich begrenzten Aufenthalt in der minimal konstruierten Lernumgebung der Wildniscamps können Lehramtsstudierende erlebnisorientierte Erfahrungen in einem auf ihr zukünftiges Berufsfeld bezogenen Bereich sammeln. Darüber hinaus können Kompetenzen für die Lehrer/innenprofessionalisierung erworben werden. Dies zeigen zum einen die Ergebnisse aus den Befragungen, dass zukünftige Lehrkräfte und Expertinnen und Experten ein breites Potenzial in der Wildnisbildung als Beitrag für eine Bildung für nachhaltige Entwicklung sowie zur Lehrer/innenprofessionalisierung sehen. Besondere Vorteile können im emotionalen Zugang, der Schulung der Teamfähigkeit sowie in der fachdidaktischen und pädagogischen Professionalisierung gesehen werden. Die Bedeutung der Dimension des Fachwissens tritt dagegen zurück, hierfür sollten die fachlichen Grundlagen in Zukunft stärker in den Begleitseminaren zu den Wildniscamps abgesichert werden. Grundsätzlich konnte festgestellt werden, dass die meisten der Lehramtsstudierenden, die im Rahmen ihres Geographiestudiums das Modul besucht haben, von dem Wildniscamp als Lernort auch nach vorheriger Skepsis - überzeugt sind. Durch den Aufenthalt im Wildniscamp dominiert besonders der Kompetenzbereich der Handlung, der bei der Thematisierung einer Bildung für nachhaltige Entwicklung auf universitärer Ebene oftmals vorwiegend auf theoretischer Ebene erfolgt. Das eigene Erleben und Reflektieren von Aspekten der Wildnisbildung, bezogen auf den Geographieunterricht, fördert die zukünftige Tätigkeit als Lehrer/in.

Grundsätzlich muss jedoch über eine Begriffserweiterung des bisher nationalparkspezifischen Bildungskonzeptes nachgedacht werden, die über bisherige Sichtweisen und Perspektiven hinausgeht. Gründe sind in den zunehmend Nationalparkgrenzen überschreitenden Aktivitäten, Wildnisflächen auch außerhalb von Großschutzgebieten einzurichten (z. B. DBU-Naturerbeflächen), zu sehen (Culmsee \& Wahmhoff 2013). Parallel sind Entwicklungstenden- zen zur Wildnisbildung auch außerhalb von Nationalparken zu verzeichnen (Bittner 2014), um einer breiten Bevölkerung, insbesondere Schüler/innen, in der Nähe der jeweiligen Lebensorte diese Bildungserfahrung zu ermöglichen.

Inwieweit die Ziele, Bedingungen und Maßnahmen der Wildnisbildung in Nationalparken auf Räume außerhalb von Großschutzgebieten übertragbar sind, muss in den nächsten Jahren erörtert werden. Das Potenzial allein der Wildnisflächen scheint vielfältig zu sein, wie z. B. Tagebaurestlöcher und -seen sowie urbane Gebiete, die keiner anthropogenen Nutzung mehr unterliegen. In einem weiteren DBUgefördertem Projekt „Wilde Nachbarschaft“ (Laufzeit: 2015-2019) sollen die Potenziale und Grenzen von Wildnisbildung außerhalb von Großschutzgebieten für unterschiedliche Zielgruppen analysiert werden. Ziel ist es, Wildnisbildung stärker in den Fokus des urbanen Lebensraums zu rücken und somit eine größere Anzahl an Lehrenden und Lernenden zu erreichen.

\section{Literatur}

Baumert, J. \& M. Kunter (2006): Stichwort: Professionelle Kompetenz von Lehrkräften. In: Zeitschrift für Erziehungswissenschaft 9 (4), 469-520.

Bibelriether, H. (1998): Faszination Wildnis - wissenschaftlich nicht erfaßte Realität. In: Nationalpark 98 (3), 4-9.

Bittner, A. (2014): Wildnisbildung - eine naturschutzfachliche wie didaktische Herausforderung. In: B. Langenhorst, A. Lude \&. A. Bittner (Hrsg.): Wildnisbildung. Neue Perspektiven für Großschutzgebiete, München: Oekom, 101-118.

BMUB - Bundesministerium für Umwelt, Naturschutz, Bau und Reaktorsicherheit (Hrsg.) (2015): Nationale Strategie zur biologischen Vielfalt (4. Aufl). http:// www.biologischevielfalt.de/fileadmin/NBS/ documents/broschuere_biolog_vielfalt_strategie_bf.pdf (31.01.2016).

BMUB - Bundesministerium für Umwelt, Naturschutz, Bau und Reaktorsicherheit \& BfN - Bundesamt für Naturschutz (Hrsg.) (2013): Naturbewusstsein 2013. Bevölkerungsumfrage zu Natur und biologischer Vielfalt. https://www.bfn.de/fileadmin/MDB/ documents/themen/gesellschaft/Naturbewusstsein/ Naturbewusstsein_2013.pdf (31.01.2016).

BUND - Freunde der Erde (2002): Wildnisbildung - ein Beitrag zur Bildungsarbeit in Nationalparken. http:// www.nationalpark-saechsische-schweiz.de/wp-content/ uploads/2013/09/Wildnisbildung.pdf (31.01.2016).

Culmsee, H. \& W. Wahmhoff (2013): Entwicklung eines leitbildorientierten Monitorings für den Naturschutz auf Flächen des Nationalen Naturerbes. In: Natur und Landschaft 88 (5), 204-212. 
DBU - Deutsche Bundesstiftung Umwelt (Hrsg.) (2013): „Wildnis vor der eigenen Haustür“: Naturerbe für die Zukunft bewahren. Pressemitteilung vom 18. Mai 2013, Nr. 42/2013. https://www.dbu.de/ media/280513015158nm0m.pdf (31.01.2016).

DGfG - Deutsche Gesellschaft für Geographie (2010): Rahmenvorgaben für die Lehrerausbildung im Fach Geographie an deutschen Universitäten und Hochschulen. Bonn: Selbstverlag Deutsche Gesellschaft für Geographie (DGfG).

DGfG - Deutsche Gesellschaft für Geographie (2014): Bildungsstandards im Fach Geographie für den Mittleren Schulabschluss mit Aufgabenbeispielen (8. Aufl.). Bonn: Selbstverlag Deutsche Gesellschaft für Geographie (DGfG).

DUK - Deutsche UNESCO-Kommission e.V. (Hrsg.) (2014): UNESCO Roadmap zur Umsetzung des Weltaktionsprogramms „Bildung für nachhaltige Entwicklung“. Deutsche Übersetzung. http://www.bneportal.de/fileadmin/unesco/de/Downloads/Dekade_ Publikationen_international/2015_Roadmap_deutsch. pdf (31.01.2016).

EU - European Union (2012): Draft Guidelines for the management of wilderness and wild areas in Natura 2000. http://ec.europa.eu/environment/nature/natura2000/ wilderness/pdf/guidance.pdf (06.04.2016).

EUROPARC Deutschland (2010): Richtlinien für die Anwendung der IUCN-Managementkategorien für Schutzgebiete. Deutsche Übersetzung (stellenweise gekürzt oder ergänzt. Berlin. https://www.bfn.de/ fileadmin/MDB/documents/themen/gebietsschutz/ IUCN_Kat_Schutzgeb_Richtl_web.pdf (12.06.2016).

Fuller, F.F. (1969). Concerns of teachers: A development conceptualization. In: American Educational Research Journal 6 (2), 207-226.

Finck, P., M. Klein \& U. Riecken (2013): Wildnisgebiete in Deutschland - von der Vision zur Umsetzung. Ergebnisse einer wissenschaftlichen Fachtagung des BfN vom 19. bis 21.11.2012 auf der Insel Vilm. In: Natur und Landschaft. Zeitschrift für Naturschutz und Landschaftspflege 88 (8), 342-346.

Gropengießer, H. (2008). Qualitative Inhaltsanalyse in der fachdidaktische Lehr-Lernforschung. In: Ph. Mayring \& M. Glaeser-Zikuda (Hrsg.): Die Praxis der Qualitativen Inhaltsanalyse. Weinheim und Basel: Beltz, 172-189.

Haan, G. de (1999): Zu den Grundlagen der „Bildung für nachhaltige Entwicklung “ in der Schule. In: Unterrichtswissenschaft 27 (3), 252-280.

Hall, G.E. \& S.M. Hord (2006): Implementing change: Patterns, principles, and potholes. Boston, MA: Pearson Education.

Hellberg-Rode, G., G. Schrüfer \& M. Hemmer (2014): Brauchen Lehrkräfte für die Umsetzung von BNE spezifische professionelle Handlungskompetenzen? Theoretische Grundlagen, Forschungsdesign und erste Ergebnisse. In: Zeitschrift für Geographiedidaktik, Journal of Geography Education 42 (4), 257-281.

Hemmer, I., P. Bagoly-Simó \& C. Fischer (2013): Koexistenz oder Kooperation? Bildung für nachhaltige Ent- wicklung an Hochschulen und Umweltbildungseinrichtungen. In: Geographie und ihre Didaktik 41 (1), 1-17.

Hottenroth, D. (2015): Professionalisierung von Lehramtsstudierenden am Beispiel Wildnisbildung. Eine explorative Längsschnittstudie. In: Wendt, P.-U. (Hrsg.): Wildnis macht stark. Marburg: Schüren Verlag, 46-68.

KMK - Kultusministerium der Länder in der Bundesrepublik Deutschland (2004). Standards für die Lehrerbildung: Bildungswissenschaften. http://www.kmk.org/fileadmin/ veroeffentlichungen_beschluesse/2004/2004_12_16Standards-Lehrerbildung.pdf (31.01.2016).

KMK - Kultusministerium der Länder in der Bundesrepublik Deutschland (2013): Ländergemeinsame inhaltliche Anforderungen für die Fachwissenschaften und Fachdidaktiken in der Lehrerbildung. http://www. $\mathrm{kmk}$.org/fileadmin/Dateien/veroeffentlichungen_ beschluesse/2008/2008_10_16-FachprofileLehrerbildung.pdf (31.01.2016).

Langenhorst, B. (2012): Was ist Wildnisbildung? http:// naju.nationalpark-kellerwald-edersee.de/de/10_ Wildnisbildung/index.php (30.04.2015).

Langenhorst, B. \& A. Lude (2014): Wirkt Wildnis? - Zur Bedeutung der Erfahrung verwilderter Natur. In: Langenhorst, B., A. Lude \& A. Bittner (Hrsg.): Wildnisbildung. Neue Perspektiven für Großschutzgebiete. München: Oekom, 213-253.

Langenhorst, B., A. Lude \& A. Bitter (Hrsg.) (2014): Wildnisbildung. Neue Perspektiven für Großschutzgebiete. München: Oekom.

LeNa - Deutschsprachiges Netzwerk LehrerInnenbildung für nachhaltige Entwicklung (2014): LehrerInnenbildung für eine nachhaltige Entwicklung - von Modellprojekten und Initiativen zu neuen Strukturen! Ein Memorandum zur Neuorientierung von LehrerInnenbildung in Deutschland, Österreich und der Schweiz. http://www.leuphana.de/fileadmin/user_upload/ PERSONALPAGES/Fakultaet_3/Stoltenberg_Ute/ Memorandum_LeNa_01_09_14.pdf (30.04.2015).

Lindau, A.-K. (2015): Das Projekt "Wildnis macht stark“ - Wildnisbildung in der universitären Geographielehrerbildung. In: Wendt, P.-U. (Hrsg.): Wildnis macht stark. Marburg: Schüren Verlag, 30-45.

Mayring, Ph. (2015): Qualitative Inhaltsanalyse: Grundlagen und Technik (12. Aufl.). Weinheim: Beltz.

Mittermeier, R., C.G. Mittermeier, P.R. Gil, G. Fonseca. T. Brooks, J. Pilgrim \& W.R. Konstant (2002): Wilderness: Earth's Last Wild Places. Conservation International. Chicago: CEMEX.

Pant, H.A., M. Vock, C. Pöhlmann \& O. Köller (2008): Eine modellbasierte Erfassung der Auseinandersetzung von Lehrkräften mit den länderübergreifenden Bildungsstandards. In: Lankes, E.M. (Hrsg.): Pädagogische Professionalität als Gegenstand empirischer Forschung. Münster: Waxmann, 245-260.

Stoltenberg, U. (2013): Potenziale des Konzepts „Bildung für eine nachhaltige Entwicklung “ für die Schulpraxis. In: Stoltenberg, U. \& V. Holz (Hrsg.): Bildung für eine nachhaltige Entwicklung in Schulen. Herausforderun- 
gen für die Lehrerbildung. Lüneburg: Nordlanddruck $\mathrm{GmbH}, 25$.

Young, J., E. Haas \& E. McGown (2014): Grundlagen der Wildnispädagogik. Mit dem Coyote-Guide zu einer tieferen Verbindung zur Natur. Buch 1 - Handbuch für Mentoren. Extertal: Biber-Verlag.
Zucchi, H. \& P. Stegmann (Hrsg.) (2006): Wagnis Wildnis. Wildnisentwicklung und Wildnisbildung in Mitteleuropa. München: Oekom Verlag. 